\title{
The Role of Regulatory B Cells in Health and Diseases: A Systemic Review
}

This article was published in the following Dove Press journal:

Journal of Inflammation Research

\author{
Endeshaw Chekol Abebe (D) \\ Tadesse Asmamaw Dejenie $\mathbb{D}^{2}$ \\ Teklie Mengie Ayele $\mathbb{D D}^{3}$ \\ Nega Dagnew Baye ${ }^{4}{ }^{4}$ \\ Assefa Agegnehu Teshome ${ }^{4}$ \\ Zelalem Tilahun Muche ${ }^{5}$
}

'Department of Biochemistry, College of Health Sciences, Debre Tabor University, Debre Tabor, Ethiopia; ${ }^{2}$ Department of Biochemistry, College of Medicine and Health Sciences, University of Gondar, Gondar, Ethiopia; ${ }^{3}$ Department of Pharmacy, College of Health Sciences, Debre Tabor University, Debre Tabor, Ethiopia; ${ }^{4}$ Department of Human

Anatomy, College of Health Sciences, Debre Tabor University, Debre Tabor, Ethiopia; ${ }^{5}$ Department of Physiology, College of Health Sciences, Debre Tabor University, Debre Tabor, Ethiopia
Correspondence: Endeshaw Chekol Abebe Department of Biochemistry, College of Health Sciences, Debre Tabor University, P. O. Box: 272; Debre Tabor Town, 6300, Ethiopia

Tel +251928428133

Email endeshawchekole@gmail.com

\begin{abstract}
Equivalent to regulatory T cells, a novel B cell populace, called regulatory B cells (Bregs), has been found to exert a negative immune regulatory role. These subsets of cells account for $0.5 \%$ of human $\mathrm{B}$ cells from the periphery that expand after activation upon certain stimuli depending on the nature of the microenvironment and provide a variety of Breg cell phenotypes. The increasing number of suppressive mechanisms attributed to Bregs suggests that these immune cells play many roles in immune regulation. Bregs have been confirmed to play a role in host defense mechanisms of healthy individuals as well as they play pathologic and protective roles in diseases or other conditions. Accumulating evidence reported that Bregs have a role in autoimmune and infectious diseases to lower inflammation, and in cancer to attenuate antitumor immune responses, thereby to promote cancer growth and metastasis. More recently, Bregs are also found to be involved in conditions like transplantation for transplant tolerance, during pregnancy to create an immune-privileged uterine environment and during early neonate life. Herein, the review summarizes recent findings aimed to provide understanding on the Breg cells, in the hope to gain insight on the general overview, development, mechanism of activation, and action of Bregs as well as their potential roles in health and diseases.
\end{abstract}

Keywords: regulatory B cells, immune regulation, health, disease

\section{Introduction}

B cells or B lymphocytes are predominantly derived from a common lymphoid progenitor in the bone marrow, where they develop from pro B cells to pre-B cells and eventually to immature B cells before they move into the periphery as transitional cells. B cells have a variety of immune functions including antigen presentation, antibody production, and direct antigen pick up through their B cell receptor (BCR). ${ }^{1,2}$ Recently, the newly designated B cells, called regulatory B cells (Breg) have been found to exert negative immunoregulatory activity. ${ }^{3}$ Breg cells, the collective name of several regulatory B cell subsets, take a very small percentage $(\approx 0.5 \%)$ of the population of B cells in healthy humans. Although the term Breg cell emerges just 17 years ago, the first description of B cells with their antiinflammatory activities dates to the 1970s. ${ }^{4}$ Bregs suppress immune-mediated inflammatory responses and/or facilitate the recovery of inflammation, thereby maintaining tolerance and immune homeostasis through production of different cytokines such as interleukin (IL)-10 and IL-35, transforming growth factor $\beta$ (TGF- $\beta$ ) and through cell contact-dependent suppressive mechanisms. ${ }^{5,6}$ This is apparent in animal models depleted of B cells, where there is worsening of the 
symptoms of inflammatory diseases including autoimmune diseases, infections, cancer, graft rejection, and other diseases, suggesting certain B cell lineages, now called Breg cells, suppress immunological disorders by preventing the expansion of pathogenic $\mathrm{T}$ cells and other inflammatory lymphocytes in such diseases. ${ }^{7,8}$

\section{Regulatory B Cell Development and Phenotypes}

The developmental stages of B cells can be classified into pre-B cells, immature B cells, mature B cells, activated B cells, and plasma cells. Within a bone marrow, the development of pre-B cells and immature B cells occurs in antigen-independent manner, while the differentiations of mature B cells, activated B cells, and plasma cells are antigen-dependent and occur in peripheral organs. ${ }^{9}$

Based on surface markers, B cells are grouped into B1 and B2 cells. B2 cells represent the majority of B cells and are commonly called B cells. B cells are further classified into follicular (FO) B cells and marginal zone (MZ) $\mathrm{B}$ cells. FO B cells are located in lymphoid follicles of secondary lymphoid tissues and the circulation whereas MZ B cells are mainly found in the marginal zone of the spleen. Both FO and MZ B cells emerge from immature precursors derived from the bone marrow. These immature B cells are called transitional B cells and depending on their maturation stage, they are divided into transitional stage 1 (T1), stage 2 (T2), and stage 3 (T3) B cells. ${ }^{9}$ FO $\mathrm{B}$ cells facilitate humoral responses to thymus-dependent antigens and enter the germinal center where they undergo class switch and affinity maturation with the aid of $T$ cells to produce antibodies of high affinity. ${ }^{10}$ Upon activation, FO and MZ B cells can undergo terminal differentiation to antibody-producing plasma cells. IgM memory cells, which express surface IgM and CD27, are considered the human equivalents of $\mathrm{MZ} \mathrm{B}$ cells. B1 cells mount humoral responses to thymus-independent antigens along with MZB cells, leading to the secretion of highly reactive lowaffinity antibodies. B1 cells are formed in the fetal liver and reside in the chest and abdominal cavity. Peritoneal B1 cells produce Mac1 (CD11b), while spleen B1 does not. $\mathrm{B} 1$ cells can be further divided into B1a (CD11b+ CD5+) and $\mathrm{B} 1 \mathrm{~b}(\mathrm{CD} 11 \mathrm{~b}+\mathrm{CD} 5-)$ cells, irrespective of the presence or absence of CD5 expression. ${ }^{9-11}$

Bregs, particularly those present in humans, have been recently characterized though there is still some controversy and unclear ideas concerning whether Breg cells are uniquely derived from a specific precursor or originate within conventional B cell subsets as well as their relationship to each other and with other B cell populations. ${ }^{10}$ Different studies report that Breg cells or their progenitors appear to be able to originate from both B1 and B2 cell subpopulations. ${ }^{9,10}$

Besides, research focused on the identification of the exact Breg subsets has yielded supporting results, which suggests Bregs do not originate from a common progenitor and that these cells may develop from various subsets of B cells. ${ }^{11}$ Bregs have a variety of sources including type T2 B cells, MZ B cells, B1b cells, and possibly FO B cells. Breg cells are grouped into a primitive type and acquired type. Primitive Bregs are mainly derived from mesenteric lymph nodes and differentiate into acquired Bregs when induced by a large number of metabolites produced by intestinal bacteria. The acquired Bregs in turn induce a series of immunosuppressive effects. ${ }^{4}$

Regarding the origin of functional Breg, numerous literatures suggest that their origin depends not only on the particular subset but also on other factors such as the $B$ cell activation status and their specific microenvironment. ${ }^{12-14}$ These studies also describe the inability to identify a specific transcription factor for Bregs as well as the heterogeneity of their phenotypes, indicate that Breg cells are not a distinct developmental line and hence any B cell can be differentiated to Bregs in response to stimuli. In response to some endogenous and exogenous stimuli, B cells can differentiate into induced Bregs (iBregs). T cells expressing cytotoxic T lymphocyteassociated protein 4 (CTLA-4) has been shown to promote the differentiation of iBreg population. The effect of $B$ cells is also highly influenced by a specific microenvironment. ${ }^{14}$ Several microenvironmental factors such as IL-21 have been demonstrated to be helpful in B cell homeostasis. IL-21 is a stimulus that drives the differentiation of $\mathrm{B}$ cells into granzyme $\mathrm{B}$ producing B cells $\left(\mathrm{GZMB}^{+}\right){ }^{12}$

Though Breg cell origin is still unclear, there are several phenotypic variants of Bregs that have been recognized in different mice models as well as in various human disease conditions. ${ }^{15,16}$ These varieties of Bregs subsets involve immature B cells, B10 cells, $\mathrm{GZMB}^{+}$B cells, regulatory $\mathrm{B} 1(\mathrm{Br} 1)$ cells, plasmablast, $\mathrm{TIM}^{+} \mathrm{B}$ cells, and iBreg cells. B cell population contains a relatively large number $\mathrm{B}$ cell subset producing a primary cytokine IL-10 called B10 cells. IL-10 is a cytokine that lowers the synthesis of proinflammatory cytokines, for instance, IL-2, 
IL-3, interferon $\gamma$ (IFN- $\gamma$ ), and tumor necrosis factor $\alpha$ (TNF- $\alpha$ ), and also inhibits the immune responses of Th1 cells. Human Breg cells are first identified as $\mathrm{CD} 19^{+} \mathrm{CD} 24^{\mathrm{hi}} \mathrm{CD} 38^{\mathrm{hi}}$ cells that accounted for the highest proportion of $\mathrm{B} 10$ cells in peripheral blood. ${ }^{15}$

\section{Mechanisms of Activation of Regulatory B Cells}

Breg cells remain at relatively low levels in normal states to maintain immunological tolerance and homeostasis while they increase in response to inflammation. Immuneactivation is required for Bregs to exhibit suppressive functions. The signals that induce the differentiation and hence the activation of Bregs include the engagement of combinations of several molecules, including toll-like receptor (TLR)-2, TLR-4, TLR-9 signaling, BCR signaling and costimulation mediated by $\mathrm{CD} 40, \mathrm{CD} 80 / \mathrm{CD} 86$ or B cell-activating factor (BAFF) and cytokines (IL-1 $\beta$, IL2, IL-6, IL-21, IL-35, IFN- $\alpha / \beta) .{ }^{17}$

$\mathrm{B}$ cells bind antigens via BCR then deliver them to cognate $\mathrm{CD} 4+\mathrm{T}$ cells and become activated with an endogenous signal CD40-CD40L, CD80/86-CD28, and IL-21, and ultimately evolve into Bregs. ${ }^{5,10,18}$ External signals like lipopolysaccharides (LPS) on TLR-2/4 and DNA complex on TLR-9 can induce Bregs by enhancing BCR signal transduction through the myeloid differentiation primary response gene 88 (Myd88) pathway to produce effector cytokines like IL-10. ${ }^{10,19}$ Cyclic AMP responsive element-binding protein (CREB) and signal transducer and activator of transcription 3 (STAT3) are the key transcription factors responsible for the expansion and differentiation of human Bregs. Besides, proinflammatory cytokines such as IL-1 $\beta$, IL-2, IL-6, and IFN- $\alpha$ help to activate Bregs via the STAT3 pathway. Moreover, Bregs are induced by anti-inflammatory cytokine IL-35 via IL-12R $\beta 2$ and IL$27 \mathrm{R} \alpha{ }^{20}$

\section{Mechanisms of Action of Regulatory B Cells}

The effector mechanisms of human Breg cells are predominantly through secretion of different anti-inflammatory cytokines, which in turn can be IL-10 dependent and IL10 independent mechanisms. ${ }^{21}$ Many functions of Breg cells have been identified to be mediated via IL-10 dependent manner by releasing their hallmark immunosuppressive cytokine called IL-10. The IL-10 derived from B cells effectively suppresses the proliferation and inflammatory cytokine production of the T cells and can also induce fork head box protein $3\left(\mathrm{FoxP}^{+}\right)$regulatory T cells. Because of its effects on innate cell types, IL-10 also acts indirectly by inhibiting antigen presentation and proinflammatory cytokine production by dendritic cells (DC), monocytes, and macrophages. ${ }^{2}$ Beyond IL-10, Bregs can act by secreting other anti-inflammatory cytokines such as TGF- $\beta$ and IL$35 .^{22}$

Furthermore, to exert their effects on target cells, Bregs can use enzymes such as intracellular granzyme B, indoleamine pyrrole 2.3-dioxygenase (IDO), or the membraneexpressed CD73 that mediates the generation of adenosine. $^{21,22}$ Granzyme B is a cytotoxic serine protease produced by Bregs, which upon activation with IL-21 and along with the engagement of BCR mediate the leakage of the enzyme into the cytoplasm that may result in the apoptotic demise of autoreactive B cells and negative immunoregulatory effects on other immune cells. Granzymes have different extracellular effects in addition to their cytotoxic functions and thus participate, for example, in inflammatory processes and tissue remodeling. There is also evidence that suggests granzyme B plays an important role in antiviral immune responses, in the regulation of autoimmune responses, and cancer immunosurveillance. ${ }^{12}$

Bregs can also exert their effects by cell contactdependent suppressive mechanisms independent of cytokine secretion. Several Breg cell surface molecules including CD80, CD86, fas ligand (FasL), programmed death ligand-1 (PD-L1), CD40L, or CD1d have been implicated in mediating suppressive function of Breg cells via direct cell-cell interactions. These molecules cause inhibition of effector $\mathrm{T}$ cell function, induction of target cell apoptosis, and induction of Treg cells. A major histocompatibility complex (MHC) class I-like molecule, called CD1d, is responsible for lipid antigens presentation to natural killer $\mathrm{T}$ (NKT) cells. CD1d is a major phenotypic marker highly expressed in many Breg cells, and it has also been suggested to have a crucial role in Breg cell-mediated suppression. The up-regulation of CD1d on B cells is associated with $\mathrm{B}$ cell-mediated protection against intestinal mucosal inflammation. ${ }^{4}$

Finally, some Bregs subsets can switch to produce immunoglobulin such as IgM and IgG4 that are related to immune-suppressive functions after activation. The human $\operatorname{IgM}$ memory cells produce $\operatorname{IgM}$ and dampen inflammatory responses by producing MyD88-dependent IL-10. ${ }^{23}$ Natural IgM are often the results of B1 cell clones 
that arise during immune development without an absolute requirement for exogenous antigenic stimulation in the mouse immune system. In murine, natural IgM antibodies that recognize apoptotic cells have been shown to increase the phagocytic clearance of dead and dying cells and to inhibit innate immune signaling pathways. Similarly, IgM autoantibodies bind to neo-epitopes on apoptotic cells in patients with systemic lupus erythematosus (SLE) and are found to be significantly higher in patients with lower disease severity and with less serious organ damage. ${ }^{24}$ Another immunoglobulin called IgG4 is recognized to be anti-inflammatory and defensive against allergic responses by competing with IgE for allergen binding. ${ }^{23}$

Collectively, there are now substantial data demonstrating that Bregs can cross-talk and suppress the functionality of immune cells of lymphoid origin by inhibiting IL-17 or IFN- $\gamma$ production in Th17, Th1, or CD8+ T cells, as well as they, suppress cells of myeloid origin by preventing the secretion of TNF- $\alpha$ or IL-12 in inflammatory monocytes or DCs, respectively. 5,22 Besides, Bregs help maintain immunological homeostasis by impairing antigen-presenting cell (APC) and inducing differentiation of Tregs and Tr1 cells (producing IL-10 and TGF $\beta$ ) as well as by activating invariant NK cells (inducing them to produce IL-4 and IL-13) both in mice and humans to favor an antiinflammatory microenvironment. ${ }^{22}$ More recently, Bregs are also known to have a relationship with a newly identified population of regulatory cells called myeloid-derived suppressor cells (MDSC). MDSC is demonstrated to be involved in enhancing the expression of inducible nitric oxide synthase (iNOS) as well as the production of nitric oxide (NO), which ameliorates inflammation by inducing IL-10 producing B cells. ${ }^{24}$ Breg cells suggested to take part earlier in an immune response and its response is shorter-lived compared to Treg response. This is because $\mathrm{B}$ cells can respond directly to naïve antigen by their BCR in contrast to $\mathrm{T}$ cells which respond to an antigen following cleavage of peptides. ${ }^{5}$

\section{Potential Roles of Regulatory B Cells in Health and Diseases}

Accumulated evidence suggests that Breg cells have been shown to play a role in host defense mechanisms of healthy individuals as well as in suppression or progression of diseases through different mechanisms. Primarily, this review discusses the current understanding regarding the intricate role of Bregs in health and diseases or conditions along with the possible Breg targeted therapeutic strategies that could pave the way for the improved therapies of various diseases.

\section{Autoimmune Diseases}

The immune-suppressive role of Breg cells in autoimmune disease is described in many models through the most well-known IL-10 dependent mechanism and/or IL10 independent manner. A plethora of studies have confirmed an inverse relationship between the number and function of Bregs and the severity of an autoimmune disease. ${ }^{14,25}$ The protective function of Bregs was originally described in the experimental autoimmune encephalomyelitis (EAE) model wherein immune regulation is mediated largely through IL-10 production. $^{14}$ Recent reports found that Bregs play a protective role in multiple sclerosis, systemic lupus erythematous, rheumatoid arthritis, type 1 diabetes mellitus, inflammatory bowel disease, and other autoimmune diseases in which strong proinflammatory Th1 and/or Th17 cells display serious deleterious effects in diseased individuals. ${ }^{25}$ This part of the review elaborates on the role of Bregs in autoimmune diseases.

\section{Multiple Sclerosis (MS)}

The protective role of $\mathrm{B}$ cells producing IL-10 is evident in the MS mice model, with B cells rapidly alleviated from disease after a short duration of paralysis, while mice with a B cell-specific IL-10 deficiency develop severe, chronic disease. $^{26}$ Importantly, following this knowledge of the immune regulatory roles of $\mathrm{B}$ cells, a new concept of MS immune pathogenesis and modern therapeutic options for MS treatment has been established.

\section{Systemic Lupus Erythematous (SLE)}

The study conducted on human Breg cells in SLE indicated that following TLR9 stimulation, Breg cells are strongly activated in IgM memory B cell subsets but their induction impaired in SLE. ${ }^{24}$ This is supported by the report of Ota and his colleagues who revealed that Breg cells induced by TLR9 from healthy controls inhibit the proliferation and IFN- $\gamma$ production of $\mathrm{T}$ cells, in contrast, such Bregs induction is significantly impaired in SLE. $^{27} \mathrm{~B}$ lymphocyte induced maturation protein 1 (Blimp-1) is found to have a critical role in Breg induction associated with the differentiation of plasma cell in a mouse model. Besides, more recent studies on SLE patients indicate that TLR9 induced Blimp-1 stimulation 
is greatly abrogated accompanied by less IL-10 production in B cells. ${ }^{24,27}$ These papers have also confirmed that Breg induction is impaired in SLE patients by Blimp-1silencing using Blimp-1 knocked down B cells. Taken together, compilation of data provides a better insight into the molecular mechanisms of Breg induction in humans, which in turn offers a novel clue to use Bregs for SLE treatment.

\section{Type I Diabetes Mellitus (TIDM)}

Beyond their pathogenic role, $\mathrm{B}$ cells have also regulatory roles in T1DM. Although B cells initiate pancreatic beta cells destruction by autoantibodies, they play a regulatory role after LPS mediated induction that in turn activates FasL expression, and TGF- $\beta$ and IL-10 production, which when adoptively transferred to non-obese diabetic (NOD) mice prevent disease occurrence. ${ }^{28,29}$ Thus, the induced Breg cells activate FasL mediated apoptosis of autoreactive T cells and dampened the APC function via TGF- $\beta$.

\section{Rheumatoid Arthritis (RA)}

Individuals with RA show a lower in percentage of IL-10 producing $\mathrm{B}$ cells in peripheral blood than patients with inactive disease or healthy individuals. The Bregexpressing PD-L1, an inhibitory receptor for CD80/86 costimulator, is also abrogated in RA. ${ }^{30}$ This study has also found a decreased proportion of $\mathrm{CD} 19^{+} \mathrm{CD}^{+} \mathrm{CD} 1 \mathrm{~d}^{\text {hi }}$ $\mathrm{B}$ cells in patients with RA when compared to control subjects, suggesting these B cells influence the disease activity of RA. Moreover, there are increased PD-L1 $B$ cells in good responder patients after the treatment of RA.

Conclusively, B cell depletion therapy has now been obsoleted due to its disappointing prognosis as a consequence of B cells' ability to interact with a vast array of other immune cells besides their antiinflammatory activity, instead, Bregs could be used as a novel therapy in autoimmune diseases.

\section{Allergic Diseases}

Breg cells, particularly Br1 cells, were found to be involved in induction and maintenance of allergen tolerance by preventing chronic inflammation in allergic diseases. Breg-mediated allergen tolerance involves IL-10-mediated suppression of T effector cells including Th2 responses, Treg induction, inhibition of DC maturation, modulation of Tfh cell responses, and production of anti-inflammatory high affinity and specificity IgG4 antibodies. ${ }^{14,23}$ These roles of Bregs are essential during allergen immunotherapy (AIT) to maintain homeostasis and this could open potential new windows for developing targeted therapies.

\section{Cancer}

B cells were traditionally best known against tumors through antibody production. ${ }^{31}$ More recently, however, it has been identified that Breg can inhibit CD4+ $\mathrm{T}$ cell stimulation or release cytokines to suppress the immune response and facilitate the progression of tumor. ${ }^{32}$ Bregs, through the production of anti-inflammatory cytokines like IL-10, can suppress diverse cell subtypes including T cells and can enhance the conversion of $\mathrm{T}$ cells to Treg cells, which in turn attenuate antitumor immune responses. Besides, Bregs have been recognized to be recruited to the tumor to get their immunosuppressive properties within the tumor bed and hence inhibit antitumor immune responses. ${ }^{33}$

There are extensively available evidence supporting the crucial role of Breg in tumor immunology. The important role of Breg in tumor immunology was originally described in lymphoma in which the Breg significantly suppresses antibody-mediated lymphoma depletion. ${ }^{32}$ Thus, Breg cells inhibit antitumor $\mathrm{T}$ effector cells through Treg induction. Leukemic B cells spontaneously secret huge amounts of IL-10 whereas the production of TNF- $\alpha$ by Breg cells increases the progression of skin carcinoma. ${ }^{33,34}$ The role of Bregs in promoting tumor development is supported by a report that shows a significantly higher proportion of circulating Bregs in patients with hepatocellular carcinoma (HCC) than healthy people, which is correlated positively with advanced stages, multiplicity and invasiveness of the tumor as well as with more recurrence of a tumor. ${ }^{35}$ This study was also revealed that human Bregs promote HCC tumor growth independent of Tregs in severe combined immune deficiency (SCID) mice and was confirmed the migration of Bregs from the blood into the tumor. Furthermore, this in vitro study explored that Bregs promote the growth and invasiveness of $\mathrm{HCC}$ by directly interacting with liver cancer cells via the CD40/CD154 signaling pathway. Additionally, tumor-evoked Bregs were reported to facilitate lung metastasis in breast cancer of a mouse model by producing TGF- $\beta$ that induces Tregs differentiation. ${ }^{36}$ This immune-suppressive property of Bregs is suggested to be through increased expression of TGF- $\beta$, PD-L1, CD86, and IL-10. ${ }^{37}$ 
The large number and impaired function of Tfh cells in non-small cell lung cancer (NSCLC), particularly in advanced stages, were reported to lead into tumor development by inducing the differentiation of immunosuppressive Breg cells. ${ }^{38}$ Currently, Breg-directed therapies that promote antitumor immunity are being developed following the increased understanding of Breg induction and function in tumor immunology as in NSCLC, which show the positive impact of the Breg on cancer therapy. ${ }^{38,39}$ These studies also added that those patients with metastatic cancer but having a greater number of $\mathrm{CD} 20^{+} \mathrm{B}$ cells in the lymph nodes have more chance of survival.

\section{Infections}

Bregs are recognized to have roles and are induced in a number of infections like bacterial, viral, and parasitic infections. This review summarizes the role of Bregs in some infectious diseases.

\section{Salmonella Infection}

In spite of their contribution to protective immunity against Salmonella, B cells exert suppression on innate host defense mechanisms to Salmonella by IL-10 derived from IL-10. ${ }^{19,40,41}$ Salmonella activates Breg cells to produce IL-10 in MyD88-dependent manner through TLR2/ TLR4 in vitro, while it enhances the rapid production of IL-10 expressing B cells in the spleen of infected mice in vivo. ${ }^{19}$ Beyond IL-10, CD19 $9^{+} \mathrm{CD} 138^{\text {hi }}$ Breg cells also suppress host immunity to Salmonella through IL-35 secretion. This finding is corroborated with a study involving a mice model with B-cell-specific IL-35 deficiency and demonstrated improved control of Salmonella infection and increased survival when compared to control mice. $^{42}$

\section{Hepatitis B Virus Infection (HBV)}

Several lines of evidence indicated that Breg contributes to the evasion of the body with HBV infection as well as hindering the elimination of such infection in an IL-10 dependent manner. ${ }^{43}$ Consistently, a study revealed that chronic HBV infected patients have expanded Breg and Treg cells and a greatly increased IL-10 concentration in the culture of peripheral blood mononuclear cells (PBMCs). ${ }^{44}$ The same paper also demonstrated a dramatically decreased percentage of Th1 cells in chronic HBV patients and was reported to have a negative correlation with Breg cells count. Another study also supports this result and demonstrates Bregs suppress HBV-specific CD8+T cell, Th1, and Th17 responses as well as convert CD4+T cells into Tregs in an IL-10 dependent mechanism. ${ }^{45}$

\section{Malaria}

In addition to antibody production in the immune response against malaria, B cells play a crucial role in immune regulation. The study conducted on Plasmodium chabaudi infected $\mathrm{C} 57 \mathrm{BL} / 6$ and $\mathrm{BALB} / \mathrm{c}$ mice shows that $\mathrm{CD} 19^{+} \mathrm{CD}^{+} \mathrm{CD} 1 \mathrm{~d}^{\text {hi }}$ cells are the well-established Breg subset though represented for less than $20 \%$ of IL-10 secreting $\mathrm{B}$ cells in both strains during infection. ${ }^{46}$ Though the roles of Breg cells during uncomplicated and complicated human malaria are yet to be further investigated, available evidence have shown that Breg cells have a beneficial role in severe malaria via preventing the worsening of inflammatory responses by cytotoxic T cells. ${ }^{47}$ Thus, for neuronal disorder due to sequestration of erythrocytes, Breg cells and anti-inflammatory cytokines, such as IL-10, are found to be important in inhibiting the neuro-pathogenesis of malaria. The same study also suggested that Breg cells may selectively bring suppressive effects on malarial immunopathology through IL10 production but appear to have little effect against the development of blood parasitemia. When IL-10 producing Breg cells are adoptively transferred to naïve mice, they could protect the animals from mortality. ${ }^{45}$ In contrast, Breg cells adoptive transfer to $\mathrm{C} 57 \mathrm{BL} / 6$ mice infected with Plasmodium chabaudi accompanied with a transient increase of parasitemia with no impact on the survival rate. $^{47}$

\section{Transplantation}

The essential role of B cells in transplantation is not only to serve as precursors of plasma cells, which produce alloantibodies and induce antibody-mediated rejection but also, a more recent finding, shows the suppressive activities of Breg play an important role in allotransplants by providing necessary cushion to the immune response against the transplant. ${ }^{48}$ Intriguingly, B cells have been reported to have a defect in immunosuppressive properties among patients with chronic antibody-mediated rejection. ${ }^{49}$ This suppressive activities of B cells are independent of IL-10, instead, GZMB, independently of the perforin component, mediates $\mathrm{T}$ cell apoptosis, and suppression of $\mathrm{T}$ cell proliferation. ${ }^{13}$ Therefore, $\mathrm{GZMB}^{+}$and the interaction of $\mathrm{B}$ cells with their $\mathrm{T}$ cell targets are the 
determinants of the inhibitory effect of B cells. The study has also shown that inhibiting TGF- $\beta$ has no effect on the immunosuppressive activities of B cells. Similarly, other studies also supporting this finding in which anti-CD45RB treatment induces strong antigen-specific tolerance dependent on the presence of B cells but independent from IL$10 .^{50,51}$ Besides, a study by Chesneau et al suggests that $\mathrm{B}$ cells have a higher absolute number of $\mathrm{GZMB}^{+} \mathrm{B}$ cells with a plasma cell-like phenotype and with dosedependent suppressive activities via GZMB pathway in blood from patients with a tolerant kidney graft. In these models, IL-10 rather counter regulates tolerance induction, and even exerts a negative impact and causes histologic lesions of rejection. Altogether, studies suggest that B cells, depending on the origin of Breg subsets, can be involved both in transplant tolerance and rejection or graft versus host disease (GVHD). ${ }^{13}$

Nowadays, like other immunological diseases, Bregs form an interesting area of research with conceivable applications in treating transplant rejection. These regulatory $\mathrm{B}$ cells may be helpful in developing a new immunomodulatory therapeutic technique to create a tolerogenic environment in transplantation. Infusing the in vitro prepared Bregs could be a novel strategy to actively induce tolerance and to treat graft rejection. ${ }^{52}$ Not at all like Tregs, there is no clinical trial utilizing Breg cells for cell treatment, in spite of the fact that it has been demonstrated effective in a few animal models. The expansion and assenting transfer of murine Bregs in transplantation models have appeared promising results though numerous steps have to be taken before this could be applied to the human beings.

\section{During Pregnancy and Early Life}

To avoid destructive immune responses against the semiallogeneic fetus during pregnancy, inflammatory responses are thought to be diminished and compensated by increased humoral responses. Thus, Breg cells are partly involved in immunosuppression to ensure immunological tolerance in the mother's womb throughout pregnancy. The associations of Breg cells with pregnancy success were first observed in mice. ${ }^{53}$ This suggests that the elevation in $\mathrm{CD}^{+} \mathrm{CD} 1 \mathrm{~d}^{+}$Breg cells is beneficial to avoid immunological abortion in pregnant mice. Indeed, the adoptive transfer of Bregs to abort prone mice enhances fetomaternal immunological tolerance by increasing Treg cells and maintaining DCs in an immature state. Similarly, a study involving rituximab drugs has corroborated the significant role of Bregs during human pregnancy. ${ }^{54}$
Another study has also supported the role of Bregs in which estrogen exert immune-inhibition during pregnancy by inducing of Breg cells' maturation to ensure the immune-privileged environment in the gravid uterus. ${ }^{55}$

Immediately after delivery, the neonatal immunity must modulate the transition from the virtually sterile womb to a planet full of antigens ensuing rapid microbial colonization of the mucosa. ${ }^{56}$ Thus, the newborn must develop and use different strategies such as avoidance of excessive responses to overcome these challenges, in part by the immunosuppressive role of Bregs. This is due to the need for tolerance that newborns are at relatively increased risk of developing severe infections though they have the ability to fight against pathogenic microbes.

$\mathrm{CD} 24{ }^{\text {hi }} \mathrm{CD} 38^{\text {hi }} \mathrm{B}$ cells (Bregs) have been found to be at an increased number in cord blood of healthy neonates and showed regulatory capacities by preventing $\mathrm{T}$ cell IFN- $\gamma$ production. $^{57}$ In line with this, another study has also demonstrated that neonatal $\mathrm{CD} 24{ }^{\text {hi }} \mathrm{CD} 38^{\text {hi }} \mathrm{B}$ cells have immunoregulatory roles. These $\mathrm{B}$ cells produce IL-10 upon stimulation, which has a suppressive activity on the production of IFN- $\gamma$ and IL-4 by T cells. Moreover, the elevated level of IL-10 secreting Bregs have been associated with good prognosis in late-onset neonatal sepsis. ${ }^{56}$

\section{Conclusion}

In conclusion, Breg cells have been recognized to play a pivotal role in down-regulation of inflammatory responses through IL-10 dependent mechanisms by producing IL-10, and a newly emerged IL-10 independent mechanisms by releasing IL-35 and TGF- $\beta$ as well as through cell contact-dependent mechanisms and production of enzymes like granzyme B, and IgG4 antibody. Breg cells suppress the differentiation of inflammatory Th1, Th17 and NKT cells as well as induce Tregs suppressing inflammation. Bregs also cross-talks with other regulatory myeloid cells like DC, MDSC, and macrophages. B cells acquire these cytokines mediated and cytokine independent suppressive activities upon differentiation into Breg cells in vivo. Taken together, these regulatory pathways play a beneficial role in health and different diseases such as autoimmune diseases, cancer, transplantation, and infectious diseases. This improved understanding of Breg cells' role may offer novel opportunities for the Breg targeted therapy. However, the molecular pathways and the subsets of B cell implicated in these various effects yet a long way to go and to be investigated thoroughly. This is because the mechanisms of Breg action have been identified in 
a disease dependent manner as the Breg is a collective name with no clearly defined phenotype, which made their study challenging. Therefore, further extensive investigation should be done to understand how they emerge and are induced to stimulate their regulatory actions before clinical application of Breg-based therapy and then after more attention should be given in designing a vaccine or therapeutic strategies against inflammatory diseases.

\section{Abbreviations}

APC, antigen presenting cells; BAFF, B-cell activating factor; BCR, B cell receptor; Blimp1, B cell induced maturation protein 1; $\mathrm{Br} 1$, regulatory $\mathrm{B} 1 \mathrm{cell}$; Breg, regulatory B cell; BTLA, B and T lymphocyte attenuators; CD40L, cluster of designation 40 ligand; CREB, cAMP responsive element binding protein; CTLA4, cytotoxic $\mathrm{T}$ lymphocyte-associated protein 4; DC, dendritic cells; EAE, experimental autoimmune encephalomyelitis; FasL, fas ligand; FO, follicular B cells; FoxP3, forkhead box protein 3; GITRL, glucocorticoid-induced tumor necrosis factor receptor-related ligand; GVHD, graft versus host disease; $\mathrm{GZMB}^{+}$, granzyme B positive; HLA, human leukocyte antigen; HSC, hematopoietic stem cell; HVEM, herpes virus entry mediator; iBreg, inducible regulatory B cells; IDO, indoleamine 2.3-dioxygenase; iNOS, inducible nitric oxide synthase; LPS, lipopolysaccharides; Mac1, macrophage1 antigen (CD11b/CD18); MDSCs, myeloid-derived suppressor cells; MHC, major histocompatibility complex; MS, multiple sclerosis; Myd88, myeloid differentiation primary response gene 88; MZB, marginal zone B cells; NKT, natural killer T cells; NOD, non-obese diabetic; NSCLC, non-small cell lung cancer; PBMCs, peripheral blood mononuclear cells; PD-1/PD$1 \mathrm{~L}$, programmed death 1 /its ligand; RA, rheumatoid arthritis; SCID, severe combined Immuno deficiency; STAT3, signal transducer and activator of transcription 3; TCR, $\mathrm{T}$ cell receptor; $\mathrm{Tfh}$, follicular helper $\mathrm{T}$ cell; $\mathrm{Th}, \mathrm{T}$ helper cell; TIM1, T cell immunoglobulin domain \& mucin domain protein 1; TLR, toll-like receptor; $\operatorname{Tr} 1$, regulatory $\mathrm{T}$ cell 1 ; Treg, regulatory $\mathrm{T}$ cell.

\section{Data Sharing Statement}

Not applicable.

\section{Ethical Approval and Consent to Participate}

Not applicable.

\section{Consent for Publication}

Not applicable.

\section{Author Contributions}

All authors made a significant contribution to the work reported, whether that is in the conception, study design, execution, acquisition of data, analysis and interpretation, or in all these areas; took part in drafting, revising or critically reviewing the article; gave final approval of the version to be published; have agreed on the journal to which the article has been submitted; and agreed to be accountable for all aspects of the work.

\section{Funding}

There is no funding to report.

\section{Disclosure}

The authors report no conflicts of interest for this work.

\section{References}

1. Kato A, Hulse KE, Tan BK, Schleimer RP. B-lymphocyte lineage cells and the respiratory system. J Allergy Clin Immunol. 2013;131 (4):933-957. doi:10.1016/j.jaci.2013.02.023

2. Moore TC, Gonzaga LM, Mather JM, Messer RJ, Hasenkrug KJ. $\mathrm{B}$ cell requirement for robust regulatory $\mathrm{T}$ cell responses to Friend retrovirus infection. MBio. 2017;8:4. doi:10.1128/mBio.01122-17

3. Han J, Sun L, Fan X, et al. Role of regulatory B cells in neuroimmunologic disorders. J Neurosci Res. 2016;94(8):693-701. doi:10.1002/ jnr.23749

4. Mizoguchi A, Mizoguchi E, Smith RN, Preffer FI, Bhan AK. Suppressive role of B cells in chronic colitis of T cell receptor $\alpha$ mutant mice. J Exp Med. 1997;186(10):1749-1756. doi:10.1084/ jem.186.10.1749

5. Rosser EC, Mauri C. Regulatory B cells: origin, phenotype, and function. Immunity. 2015;42(4):607-612. doi:10.1016/j. immuni.2015.04.005

6. Harris DP, Haynes L, Sayles PC, et al. Reciprocal regulation of polarized cytokine production by effector $\mathrm{B}$ and $\mathrm{T}$ cells. Nat Immunol. 2000;1(6):475-482. doi:10.1038/82717

7. Shlomchik MJ, Craft JE, Mamula MJ. From $T$ to B and back again: positive feedback in systemic autoimmune disease. Nat Rev Immunol. 2001;1(2):147-153. doi:10.1038/35100573

8. Xiao X, Lao X-M, Chen -M-M, et al. PD-1hi identifies a novel regulatory B-cell population in human hepatoma that promotes disease progression. Cancer Discov. 2016;6(5):546-559. doi:10.1158/ 2159-8290.CD-15-1408

9. Yanaba K, Bouaziz J-D, Matsushita T, Tsubata T, Tedder TF. The development and function of regulatory B cells expressing IL-10 (B10 cells) requires antigen receptor diversity and TLR signals. $J \quad$ Immunol. 2009;182(12):7459-7472. doi:10.4049/jimmu nol.0900270

10. Bankó Z, Pozsgay J, Szili D, et al. Induction and differentiation of IL-10-producing regulatory B cells from healthy blood donors and rheumatoid arthritis patients. J Immunol. 2017;198(4):1512-1520. doi:10.4049/jimmunol.1600218

11. Vitale G, Mion F, Pucillo C. Regulatory B cells: evidence, developmental origin and population diversity. Mol Immunol. 2010;48(1-3):1-8. doi:10.1016/j.molimm.2010.09.010 
12. Lindner S, Dahlke K, Sontheimer K, et al. Interleukin 21-induced granzyme B-expressing B cells infiltrate tumors and regulate $\mathrm{T}$ cells. Cancer Res. 2013;73(8):2468-2479. doi:10.1158/0008-5472.CAN$12-3450$

13. Chesneau M, Michel L, Dugast E, et al. Tolerant kidney transplant patients produce B cells with regulatory properties. $J$ Am Society Nephrol. 2015;26(10):2588-2598. doi:10.1681/ASN.2014040404

14. Fillatreau S, Sweenie CH, McGeachy MJ, Gray D, Anderton SM. B cells regulate autoimmunity by provision of IL-10. Nat Immunol. 2002;3(10):944-950. doi:10.1038/ni833

15. Blair PA, Noreña LY, Flores-Borja F, et al. CD19+ CD24hiCD38hi $\mathrm{B}$ cells exhibit regulatory capacity in healthy individuals but are functionally impaired in systemic lupus erythematosus patients. Immunity. 2010;32(1):129-140. doi:10.1016/j.immuni.2009.11.009

16. Moritoki Y, Zhang W, Tsuneyama K, et al. B cells suppress the inflammatory response in a mouse model of primary biliary cirrhosis. Gastroenterology. 2009;136(3):1037-1047. doi:10.1053/j. gastro.2008.11.035

17. Baba Y, Matsumoto M, Kurosaki T. Signals controlling the development and activity of regulatory B-lineage cells. Int Immunol. 2015;27 (10):487-493. doi:10.1093/intimm/dxv027

18. Yoshizaki A, Miyagaki T, DiLillo DJ, et al. Regulatory B cells control T-cell autoimmunity through IL-21-dependent cognate interactions. Nature. 2012;491(7423):264-268. doi:10.1038/nature11501

19. Neves P, Lampropoulou V, Calderon-Gomez E, et al. Signaling via the MyD88 adaptor protein in B cells suppresses protective immunity during Salmonella typhimurium infection. Immunity. 2010;33 (5):777-790. doi:10.1016/j.immuni.2010.10.016

20. Hua C, Audo R, Yeremenko N, et al. A proliferation inducing ligand (APRIL) promotes IL-10 production and regulatory functions of human B cells. J Autoimmun. 2016;73:64-72. doi:10.1016/j.jaut.2016.06.002

21. Nouel A, Pochard P, Simon Q, et al. B-Cells induce regulatory T cells through TGF- $\beta /$ IDO production in A CTLA-4 dependent manner. J Autoimmun. 2015;59:53-60. doi:10.1016/j.jaut.2015.02.004

22. Ray A, Wang L, Dittel BN. IL-10-independent regulatory B-cell subsets and mechanisms of action. Int Immunol. 2015;27 (10):531-536. doi:10.1093/intimm/dxv033

23. van de Veen W, Stanic B, Wirz OF, Jansen K, Globinska A, Akdis M. Role of regulatory $\mathrm{B}$ cells in immune tolerance to allergens and beyond. $J$ Allergy Clin Immunol. 2016;138(3):654-665. doi:10.1016/j.jaci.2016.07.006

24. Miyagaki T, Fujimoto M, Sato S. Regulatory B cells in human inflammatory and autoimmune diseases: from mouse models to clinical research. Int Immunol. 2015;27(10):495-504. doi:10.1093/ intimm/dxv026

25. Moudgil KD, Choubey D. Cytokines in autoimmunity: role in induction, regulation, and treatment. J Interferon Cytokine Res. 2011;31 (10):695-703. doi:10.1089/jir.2011.0065

26. Knippenberg S, Peelen E, Smolders J, et al. Reduction in IL-10 producing B cells (Breg) in multiple sclerosis is accompanied by a reduced naive/memory Breg ratio during a relapse but not in remission. J Neuroimmunol. 2011;239(1-2):80-86. doi:10.1016/j. jneuroim.2011.08.019

27. Ota S-I, Niiro H, Ueki N, et al. Elucidation of molecular mechanisms of Breg induction in autoimmune diseases.: 1952. Arthritis Rheumatol. 2014;66.

28. Felton JL, Maseda D, Bonami RH, Hulbert C, Thomas JW. Antiinsulin $\mathrm{B}$ cells are poised for antigen presentation in type 1 diabetes. J Immunol. 2018;201(3):861-873. doi:10.4049/jimmunol.1701717

29. Tian J, Zekzer D, Hanssen L, Lu Y, Olcott A, Kaufman DL. Lipopolysaccharide-activated B cells down-regulate Th1 immunity and prevent autoimmune diabetes in nonobese diabetic mice J Immunol. 2001;167(2):1081-1089. doi:10.4049/jimmunol.167.2.1081

30. Cui D, Zhang L, Chen J, et al. Changes in regulatory B cells and their relationship with rheumatoid arthritis disease activity. Clin Exp Med. 2015;15(3):285-292. doi:10.1007/s10238-014-0310-9
31. Schneider C, Teufel A, Yevsa T, et al. Adaptive immunity suppresses formation and progression of diethylnitrosamine-induced liver cancer. Gut. 2012;61(12):1733-1743. doi:10.1136/gutjnl-2011-301116

32. Horikawa M, Minard-Colin V, Matsushita T, Tedder TF. Regulatory B cell production of IL-10 inhibits lymphoma depletion during CD20 immunotherapy in mice. J Clin Invest. 2011;121:11. doi:10.1172/ JCI59266

33. Schioppa T, Moore R, Thompson RG, et al. B regulatory cells and the tumor-promoting actions of TNF- $\alpha$ during squamous carcinogenesis. Proc Nat Acad Sci. 2011;108(26):10662-10667. doi:10.1073/ pnas. 1100994108

34. Wang X, Yuling H, Yanping J, et al. CCL19 and CXCL13 synergistically regulate interaction between $\mathrm{B}$ cell acute lymphocytic leukemia CD23+ CD5+ B Cells and CD8+ T cells. J Immunol. 2007;179 (5):2880-2888. doi:10.4049/jimmunol.179.5.2880

35. Shao Y, CM L, Ling CC, et al. Regulatory B cells accelerate hepatocellular carcinoma progression via CD40/CD154 signaling pathway. Cancer Lett. 2014;355(2):264-272. doi:10.1016/j.canlet.2014.09.026

36. Olkhanud PB, Damdinsuren B, Bodogai M, et al. Tumor-evoked regulatory $\mathrm{B}$ cells promote breast cancer metastasis by converting resting CD4+ T cells to T-regulatory cells. Cancer Res. 2011;71 (10):3505-3515. doi:10.1158/0008-5472.CAN-10-4316

37. Wejksza K, Lee-Chang C, Bodogai M, et al. Cancer-produced metabolites of 5-lipoxygenase induce tumor-evoked regulatory B cells via peroxisome proliferator-activated receptor $\alpha$. J Immunol. 2013;190 (6):2575-2584. doi:10.4049/jimmunol.1201920

38. Qiu L, Yu Q, Zhou Y, et al. Functionally impaired follicular helper $\mathrm{T}$ cells induce regulatory $\mathrm{B}$ cells and $\mathrm{CD} 14+$ human leukocyte antigen-DR- cell differentiation in non-small cell lung cancer. Cancer Sci. 2018;109(12):3751-3761. doi:10.1111/cas.13836

39. Pretscher D, Distel LV, Grabenbauer GG, Wittlinger M, Buettner M, Niedobitek G. Distribution of immune cells in head and neck cancer: CD8+ T-cells and CD20+ B-cells in metastatic lymph nodes are associated with favourable outcome in patients with oro-and hypopharyngeal carcinoma. BMC Cancer. 2009;9(1):292. doi:10.1186/ 1471-2407-9-292

40. Galdiero M, Pisciotta M, Galdiero E, Carratelli CR. Porins and lipopolysaccharide from Salmonella typhimurium regulate the expression of CD80 and CD86 molecules on B cells and macrophages but not CD28 and CD152 on T cells. Clin Microbiol Infection. 2003;9(11):1104-1111. doi:10.1046/j.14690691.2003.00728.x

41. Barr TA, Brown S, Mastroeni P, Gray D. TLR and B cell receptor signals to B cells differentially program primary and memory Th1 responses to Salmonella enterica. $J$ Immunol. 2010;185 (5):2783-2789. doi:10.4049/jimmunol.1001431

42. Shen P, Roch T, Lampropoulou V, et al. IL-35-producing B cells are critical regulators of immunity during autoimmune and infectious diseases. Nature. 2014;507(7492):366-370. doi:10.1038/nature12979

43. Gong Y, Zhao C, Zhao P, et al. Role of IL-10-producing regulatory B cells in chronic hepatitis B virus infection. Dig Dis Sci. 2015;60 (5):1308-1314. doi:10.1007/s10620-014-3358-1

44. Das A, Ellis G, Pallant C, et al. IL-10-producing regulatory B cells in the pathogenesis of chronic hepatitis B virus infection. J Immunol. 2012;189(8):3925-3935. doi:10.4049/jimmunol.1103139

45. Liu Y, Cheng L-S, Wu S-D, et al. IL-10-producing regulatory B-cells suppressed effector T-cells but enhanced regulatory T-cells in chronic HBV infection. Clin Sci. 2016;130(11):907-919. doi:10.1042/ CS20160069

46. Han X, Yang J, Zhang Y, et al. Potential role for regulatory B cells as a major source of interleukin-10 in spleen from Plasmodium chabaudi-infected mice. Infect Immun. 2018;86:5. doi:10.1128/ IAI.00016-18

47. Liu Y, Chen Y, Li Z, et al. Role of IL-10-producing regulatory B cells in control of cerebral malaria in P lasmodium berghei infected mice. Eur J Immunol. 2013;43(11):2907-2918. doi:10.1002/eji.201343512 
48. Silva HM, Takenaka MC, Moraes-Vieira PM, et al. Preserving the B-cell compartment favors operational tolerance in human renal transplantation. Mol Med. 2012;18(5):733-743. doi:10.2119/ molmed.2011.00281

49. Nouël A, Ségalen I, Jamin C, et al. B cells display an abnormal distribution and an impaired suppressive function in patients with chronic antibody-mediated rejection. Kidney Int. 2014;85 (3):590-599. doi:10.1038/ki.2013.457

50. Deng S, Moore DJ, Huang X, et al. Cutting edge: transplant tolerance induced by anti-CD45RB requires B lymphocytes. J Immunol. 2007;178(10):6028-6032. doi:10.4049/jimmunol.178.10.6028

51. Singh A, Carson WF, Secor ER, et al. Regulatory role of B cells in a murine model of allergic airway disease. J Immunol. 2008;180 (11):7318-7326. doi:10.4049/jimmunol.180.11.7318

52. Geissler EK, Hutchinson JA. Cell therapy as a strategy to minimize maintenance immunosuppression in solid organ transplant recipients. Curr Opin Organ Transplant. 2013;18(4):408-415. doi:10.1097/ MOT.0b013e328363319d
53. Jensen F, Muzzio D, Soldati R, Fest S, Zenclussen AC. Regulatory B10 cells restore pregnancy tolerance in a mouse model. Biol Reprod. 2013;89(4):90,1-7. doi:10.1095/biolreprod.113.110791

54. Chakravarty EF, Murray ER, Kelman A, Farmer P. Pregnancy outcomes after maternal exposure to rituximab. Blood, J Am Soc Hematol. 2011;117(5):1499-1506.

55. Muzzio DO, Soldati R, Ehrhardt J, et al. B cell development undergoes profound modifications and adaptations during pregnancy in mice. Biol Reprod. 2014;91(5):115,1-11. doi:10.1095/ biolreprod.114.122366

56. Li S, Ma F, Hao H, et al. Marked elevation of circulating CD19+ CD38hiCD24hi transitional B cells give protection against neonatal sepsis. Pediatr Neonatol. 2018;59(3):296-304. doi:10.1016/j. pedneo.2017.10.005

57. Sarvaria A, Basar R, Mehta RS, et al. IL-10+ regulatory B cells are enriched in cord blood and may protect against cGVHD after cord blood transplantation. Blood, J Am Soc Hematol. 2016;128 (10):1346-1361
Journal of Inflammation Research

\section{Publish your work in this journal}

The Journal of Inflammation Research is an international, peerreviewed open-access journal that welcomes laboratory and clinical findings on the molecular basis, cell biology and pharmacology of inflammation including original research, reviews, symposium reports, hypothesis formation and commentaries on: acute/chronic inflammation; mediators of inflammation; cellular processes; molecular
Dovepress

mechanisms; pharmacology and novel anti-inflammatory drugs; clinical conditions involving inflammation. The manuscript management system is completely online and includes a very quick and fair peerreview system. Visit http://www.dovepress.com/testimonials.php to read real quotes from published authors. 